\title{
Natural Hypoxia is Not a Limiting Factor in Evaluating the Novel Arylidene Derivative MLT-401 Against an In Vitro Colorectal Cancer Model
}

\author{
Ismaeel Bin-Jaliah \\ Department of Physiology, College of Medicine, King Khalid University, Abha, Saudi Arabia
}

\section{Key Words}

Colorectal carcinoma $\bullet$ Arylidene derivatives $\bullet$ Natural hypoxia $•$ Anti-cancer drugs $\bullet$ Apoptosis

\begin{abstract}
Background/Aims: Cancer cells in vivo develop resistance to many anti-tumor drugs. One known factor to influence such drug resistance is hypoxia, which is an important component of the tumor microenvironment. Standard cancer lines mostly do not exhibit a cellular hypoxic microenvironment and there is a paucity of information on the efficacy of lead molecules in both cellular- and environment-induced hypoxic conditions. Therefore, in the present study, we have evaluated the efficacy of the arylidene derivative MLT-401, a lead molecule showing activity against colorectal cancer model using the HCT 116 cell line and CCD-80-C control cells in normoxic and natural (marginal) hypoxic conditions, which is usually observed in highaltitude regions. Methods: The efficacy of MLT-401 on HCT 116 and CCD-80-C cells were tested in both normoxia and marginal hypoxia conditions. MTT assay was used to evaluate cell proliferation, Annexin $\mathrm{V}$ binding assay for apoptotic cell quantification and PI staining for cell cycle were done by flow cytometry. Induction of pro-apoptotic marker BAX and anti-apoptotic $\mathrm{BCl}-2$ were assessed by western blot. Bcl-2/BAX ratio was calculated based on protein expression by western blotting and bands were quantified by Image J software. Results: Analysis of cell proliferation showed an average 10 -fold reduction in the inhibition of HCT 116 cells in hypoxic conditions with approximately 500 nM MLT-401, while there was no significant change noted in marginal hypoxic conditions. A proportionate increase in the number of apoptotic cells and large M4 fraction of $10.5 \%$ and $26.7 \%$ of HCT116 against $6.3 \%$ of control cells in cell cycle assessment with MLT-401 concentrations ranging from 250 to 500 $\mathrm{nM}$ respectively clearly demonstrated anti-cancer activity. A Bcl-2/BAX ratio of $<1$ showed that the induction of apoptosis was the gross mechanism underlying the inhibition of HCT 116 cells by MLT-401. Conclusion: Collectively, these results show MLT-401 as an effective anticolorectal cancer lead molecule irrespective of normoxia or natural hypoxia.
\end{abstract}




\section{Cellular Physiology Cell Physiol Biochem 2018;46:2082-2089 \\ and Biochemistry Published online: May 03, $2018 \quad \begin{aligned} & \text { DOI: 10.1159/000489448 } \\ & \begin{array}{l}\text { C) } 2018 \text { The Author(s). Published by S. Karger AG, Basel } \\ \text { www.karger.com/cpb }\end{array}\end{aligned}$ \\ Bin-Jaliah: Hypoxia Effects on MLT-401 Anticancer Properties}

\section{Introduction}

Anti-cancer or anti-tumor drug activity is typically evaluated using in vitro screening techniques employing primary cells and commercial cell lines. These cell lines are standardized to laboratory conditions and often do not address the abnormal physiological nature of tissues or cells as observed in in vivo conditions [1, 2]. Hypoxia, immune evasion, extracellular matrix composition/organization, inflammation, and immunosuppressive tumor-associated cells are important physiological factors that can affect drug interactions with tumor cells and promote tumor progression [3, 4]. Some of the factors are systemic while few factors are at cellular level influenced by the physiological environment prevailing at the niche. These physiological imbalances result in the formation of a gross cellular catastrophe known as the tumor microenvironment, which has complex characteristics that are not observed in modified cell lines. Moreover, cancer progression is mostly dependent upon such persistent microenvironment and cellular deregulation which forms the basis of most important cancer microenvironment leading to drug resistance.

Lead molecules with anticancer/tumor activity are usually evaluated using standard cancerous lines that do not represent real cancer models. Although these lines do not exhibit the exact tumor microenvironment as observed in vivo, ease to use and high throughput adaptations makes it comfortable for many drug screening laboratories. In many cases, lead molecules with positive results against cancer or tumor cell lines do not translate into successful animal or clinical trials [5]. One of the limiting factors affecting drug efficacy, for in vivo tumors, is hypoxia, which is a common phenomenon observed in the tumor microenvironment and is known to influence tumor metastasis and confer drug resistance [6]. Theoretically, low oxygen availability is an important factor that can alter the therapeutic effectiveness of a drug. Further, hypoxia can induce or mitigate drug-induced toxicity and alter cellular metabolism, enzyme kinetics, and overall bioenergetic turnover of cells [7]. However, in the case of cancer drug development, the process is reversed, as the tumor microenvironment itself is hypoxic and environmental hypoxia may have its own effect on drug action [8]. Further, in vivo hypoxia is known to promote tumor cell immune evasion, a strategy that allows tumor cells to escape immune surveillance and survive. Hence, most of the lead drug molecules in vitro largely do not work in the microenvironment of a tumor. The traditional understanding of hypoxia and the cancer microenvironment is that hypoxic stress impairs tumor cell killing mediated by both innate and adaptive immune cells, which have altogether different drug interactions $[9,10]$. It is also known that hypoxic stress can also play a major role in the acquisition of anti-tumor drug resistance, which is not clearly understood. The known mechanism is that tumor cells become adapted to hypoxic microenvironment by transcriptional regulation of the hypoxia-inducible factor (HIF) family of proteins [11]. Further, the hypoxia-mediated nuclear translocation of HIF heterodimers results in the transcriptional activation of downstream targets like glucose transporter 1 (GLUT1), and CAIX that are involved in chemoresistance [12]. Hypoxia-induced chemoresistance has been discussed in the context of various molecular signals such as nuclear factor kappa-B, cyclooxygenase-2, and apoptosis inhibitory protein. Further, environmental or physiological hypoxia might alter gross cellular metabolism and may be one of the factors underlying the inductionof drug resistance [13]. As an example, hypoxia-mediated HIF proteins induce some drug-metabolizing enzymes of the cytochrome P-450 class, rendering themselves as important cofactors in cancer expression and metastatic potential [14].

To overcome these limitations of anti-tumor drugs, especially in the industrial screening where large arrays of chemicals, semisynthetic molecules and natural products are used the activity of many promising lead molecules needs to be assessed in hypoxic conditions. To address these physiological concerns, the current study was designed to use the arylidene derivative MLT-401, an indanone compound known for its biologic activity, with special reference to its anti-tumor/cancer efficacy on various cancer models $[15,16]$. The selection of MLT-401 was based on its previously reported structural similarity with arylidene indanone molecules having profound anti-tumor activity [17]. Further, these indanone compounds 


\section{Cellular Physiology Cell Physiol Biochem 2018;46:2082-2089

 \\ Bin-Jaliah: Hypoxia Effects on MLT-401 Anticancer Properties}

have not been affected by the drug resistance as observed with many other cancer drugs. Considering these facts, the anti-tumor activity of MLT-401 was evaluated in the standard colorectal cancer cell line HCT 116 and control cells CCD-80-Co. This study will also throw light on the benefits and disadvantages of natural hypoxia on the efficacy of indanone-based drugs. The preliminary results of this study were presented previously in an abstract form by the author, as a recipient of the American Physiological Society Travel Award, at the $38^{\text {th }}$ Congress of the International Union of Physiological Sciences, which was hosted by the Brazilian Physiological Society in Rio de Janeiro, Brazil [18].

\section{Materials and Methods}

\section{Cell culture}

HCT 116 and CCD-18-Co cells were grown in RPMI-1640 medium supplemented with $10 \%$ fetal bovine serum (FBS), $100 \mathrm{U} / \mathrm{mL}$ penicillin, and $100 \mathrm{U} / \mathrm{mL}$ streptomycin. All cells (passages 3-12), were maintained at $37^{\circ} \mathrm{C}$ in a $5 \% \mathrm{CO}_{2}$ environment in a humidified atmosphere until the required confluence $(70 \%)$ was attained. The medium was replaced every 2 days and maintenance was strictly in accordance with the standard methods. All experiments were performed after the due approval of the Biomedical Research Ethics Committee at King Khalid University, Abha, Saudi Arabia (REC. No. 2017-01-01).

\section{Induction of transient hypoxia}

To induce hypoxia, cell culture medium was bubbled for $30 \mathrm{~min}$ with $100 \% \mathrm{~N}_{2}$ followed by the addition of a freshly prepared sodium dithionite solution to give a final concentration of $0.25 \mathrm{mM}$, which was sufficient to reduce $\mathrm{pO}_{2}$ to low levels. The tissue culture plates were sealed with Vaseline to avoid the influence of the regular $5 \% \mathrm{CO}_{2}$ atmosphere of the incubator. This method was modified from the standard cited literature to suit our study purpose [19].

\section{Cell proliferation assay}

Cell proliferation was assessed as per our standard protocol described elsewhere [20]. Briefly, 5000 cells/well in $100 \mu \mathrm{L}$ RPMI medium supplemented with $10 \%$ FBS and $1 \%$ penicillin/streptomycin were plated in triplicates in a 96-well plate. After an overnight incubation, the medium was replenished with either fresh growth medium for normoxic conditions or low-oxygen medium for marginal hypoxic conditions containing $50 \mu \mathrm{L}$ MLT-401 to the desired final concentration. The hypoxic medium plates were sealed to avoid the external influence of gases. Dimethyl sulfoxide (DMSO) and blank controls were also included simultaneously. The medium was changed to regular fresh medium and the plates were incubated at $37^{\circ} \mathrm{C}$ and $5 \% \mathrm{CO}_{2}$ for $48 \mathrm{~h}$. Then, $15 \mu \mathrm{L}$ of $5 \mathrm{mg} / \mathrm{mL}$ MTT was added and incubated for $3.5 \mathrm{~h}$. The medium was aspirated and MTT was dissolved in $150 \mu \mathrm{L}$ DMSO and absorbance was read at $560 \mathrm{~nm}$ with reference at $640 \mathrm{~nm}$. Percentage inhibition was calculated after subtracting the value for Day 0 MTT. The results were analyzed using GraphPad Prism software.

\section{Annexin V assay}

An annexin $V$ binding assay was performed using an Annexin V Detection Kit (e-Bioscience, San Diego, CA) as per the manufacturer's instructions. Briefly, $0.5 \times 10^{6}$ cells were grown in 6-well tissue culture plates for $24 \mathrm{~h}$ until the cell sheets were semi-confluent. The cells were incubated with medium containing the final concentrations of MLT-401 used in this study. The plates were incubated in a $\mathrm{CO}_{2}$ incubator for $72 \mathrm{~h}$. Post-incubation, the cells were harvested, washed twice with wash buffer, and incubated with $0.25 \mu \mathrm{g} / \mathrm{mL}$ annexin $V$ reagent in $1 \times$ binding buffer for $15 \mathrm{~min}$. The cells were washed twice to remove excess annexin $\mathrm{V}$ and were re-suspended in binding buffer containing $0.5 \mu \mathrm{g} / \mathrm{mL}$ propidium iodide. A flow cytometer was used to acquire 10, 000 events. Early and late phase apoptotic cells were segregated with a quadri plot graph and the total percentage of apoptotic cells was presented using a bar chart.

Cell cycle analysis

Briefly, $0.5 \times 10^{6}$ cells per well were added to a 6-well tissue culture plate. MLT-401 was added at the desired concentration to the medium and the cells were incubated for $72 \mathrm{~h}$ at $37^{\circ} \mathrm{C}$ in a $5 \% \mathrm{CO}_{2}$ incubator. 
After $72 \mathrm{~h}$, the cells were fixed in $70 \%$ ethanol and stored at $-20^{\circ} \mathrm{C}$ till acquisition using a flowcytometer; 10 , 000 events were acquired and \% percentage of cell population in different cell cycle stages with respect to control was calculated [21].

\section{Western immunoblotting}

HCT 116 and CCD-18-Co cells were cultured with various concentrations of MLT-401 for $48 \mathrm{~h}$. The cells were scraped and lysed in RIPA buffer, as described elsewhere. The insoluble materials were removed by centrifugation at $14,000 \times g$ for $10 \mathrm{~min}$ at $4^{\circ} \mathrm{C}$. Protein quantification was performed using a standard Coomassie Plus Protein Assay Reagent Kit (Pierce, Rockford, IL). Cell lysate (20 $\mu \mathrm{g} / \mathrm{lane}$ ) was resolved by 8-15\% sodium dodecyl sulfate polyacrylamide gel electrophoresis followed by polyvinylidene difluoride membrane transfer. The transferred membrane was probed with anti-BAX, anti-Bcl-2, and anti- $\beta$-actin primary antibodies (Cell Signaling Technologies, Beverly, MA), followed by the addition of the corresponding horseradish peroxidase-conjugated secondary antibodies. Protein bands were visualized by using ECL reagents (Amersham Bioscience, Piscataway, NJ) and exposed to Kodak X-Omat Blue XB-1 films (Rochester, NY). Bands were quantified using Image (Ver. 1.46; NIH, Bethesda, MD) and normalized to actin and the ratio was determined.

\section{Statistical analysis}

Data from multiple repeat experiments were quantified and displayed as the mean \pm standard error of the mean. The difference between two groups was analyzed using a two-tailed Student's t test. A P-value $<0.05$ was considered significant. Statistical analyses were performed using GraphPad Prism 6.0 (La Jolla, CA).

\section{Results}

MLT-401 is a derivative of arylidene indanone compounds. MLT-401 has a molecular weight of 248.32 with a formula $\mathrm{C}_{18} \mathrm{H}_{16} \mathrm{O}$. Its structure is similar to other arylidene molecules with modifications at the positions near the arylidene ring (Fig. 1). The nontoxic dose of arylidene in the normal control CCD-18-Co cells was determined in both normoxic and marginal hypoxic conditions. The nontoxic dose of the solvent DMSO has been determined previously and is not shown here. The nontoxic concentration of MLT-401 used in the current investigation ranged from 250 to $500 \mathrm{nM}$ for HCT116 cells, while the normal CCD-18-Co cells tolerated approximately 5000 nMMLT-401 (Fig. 2a and $b$ ).

The results of the initial cell proliferation inhibition assays with various concentrations of MLT-401 showed a dose-dependent reduction in the proliferation of HCT116 colorectal carcinoma cells, while the normal CCD-18Co cells were not inhibited (Fig. 2a). Drug activity under the influence of marginal hypoxia also did not affect the inhibition of HCT116 (Fig. 2b). An average 10-fold reduction in proliferation was observed

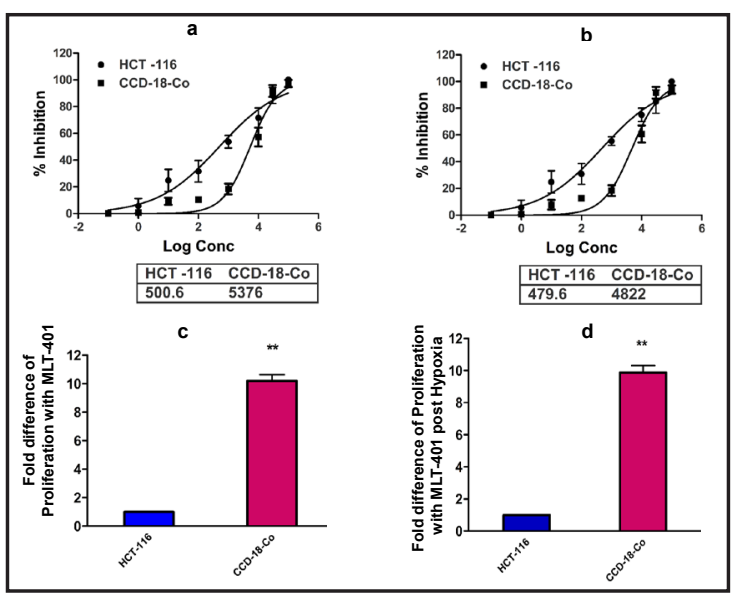

Fig. 2. Proliferation of CCD-18-Co and HCT 116 cells under (a) normoxic and (b) hypoxic conditions in the presence of MLT-401. Fold difference in the inhibition of proliferation by MLT-401 under (c) normoxic and (d) hypoxic conditions. 
with a concentration of 500 nM MLT-401 in both normal and marginal hypoxic conditions. We next investigated the basic mechanism of cell death. Our cell cycle assessment showed the accumulation of early and late phase apoptotic cells (Fig. $3 a$ and $b$ ). These results clearly indicate that MLT-401 has profound activity in inhibiting cancer in a classical manner with early and late phase apoptosis. We did not observe a similar effect with the CCD-18-Co cells. To substantiate the above observation, classical annexin $\mathrm{V}$ versus propidium iodide staining was used to assess the cell cycle. A large accumulation of M4 fraction about $10.5 \%$ to $26.7 \%$ in HCT 116 cells against $5.9 \%$ to $6.3 \%$ of control cells was observed at a concentration ranging from 250 to 500 nM MLT-401, respectively (Fig. 3c). Quantitation of the M4 fraction showed a dose-dependent increase of apoptotic cells in the HCT 116 cell line compared with the control CCD-18-Co cells.

Next, we investigated whether MLT401 inhibition of cancer cells is through the upregulation of pro-apoptotic factors. This was in lieu of the accumulation of the M4 fraction observed previously (Fig. $3 a, b$, and c). A marginal increase of Bax expression was observed in HCT 116 cells treated with 250 and 500 nM MLT401 , while a drastic reduction in $\mathrm{Bcl}-2$ expression confirmed the induction of apoptosis (Fig. 4a). Further, a Bcl-2/Bax ratio of $<1$ (Fig. 4 b) indicated apoptosis as the mode of cell death.

From the above observations, it is evident that MLT-401 inhibits the proliferation of cancer cells through the



Fig. 3. (a) Annexin V staining showing the accumulation of early and late apoptotic HCT116 cells and CCD-18Co cells after treatment with MLT-401. (b) Quantitation of the early and late apoptotic cells. (c) Cell cycle assessment of HCT116 cells showing the accumulation of the M4 phase.

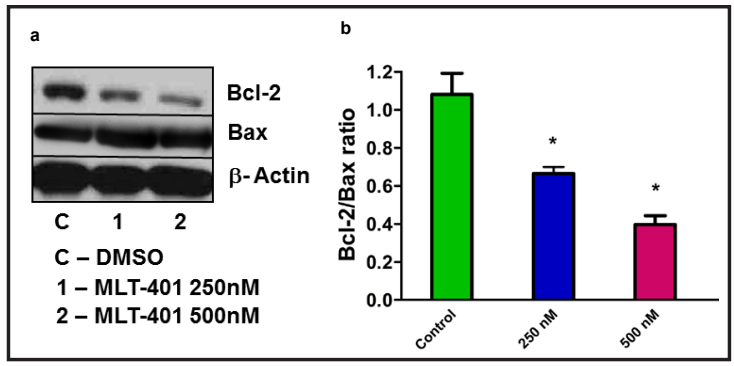

Fig. 4. (a) Western blot showing the reduction of the expression of the anti-apoptotic marker Bcl-2 following treatment with MLT-401 in HCT116 cells.

(b) Quantitation of the bands showing the reduced expression of Bcl-2. induction of apoptosis and cell cycle regulation. Further, from the results obtained, it can be hypothesized that the Bcl-1/Bax ratio of $<1$ not only indicates apoptosis but also the down-regulation of anti-apoptotic factors.

\section{Discussion}

The use of arylidene and its derivatives as candidate molecules for anti-cancer or antitumor therapy has been examined for a few decades. Arylidene rings are reported to confer stability to the molecule and its biological activity [22]. Many studies have assessed various other arylidene derivatives exhibiting antibacterial [23], antifungal [24], or anticancer [25, $26]$ activities. Other members of our study group have also reported anti-cancer properties $[17,21]$ for a few derivatives of this family.

The results of the current study show that a low concentration of MLT-401 inhibited the proliferation of HCT116 cells. Generally, a low concentration of drug inhibiting cancer cell 


\section{Cellular Physiology Cell Physiol Biochem 2018;46:2082-2089

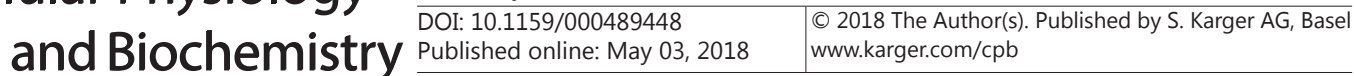 \\ Bin-Jaliah: Hypoxia Effects on MLT-401 Anticancer Properties}

proliferation may be a positive signal for a wide therapeutic window with a speculation that this might be less toxic to the normal surrounding tissues in vivo. This is evident from the results of CCD-16-Co cells that tolerated approximately 5000 nM MLT-401. Similar effects were observed with other arylidene derivatives studied using various cancer models [9, 27]. The selective inhibition of cancerous cells over normal cells both in normoxic and marginal hypoxic conditions shows that the molecule works downstream of the hypoxic conditions. Some arylidene derivatives have been shown previously to work on upstream pathways, such as the involvement of the Akt pathways ras and p53 [17, 28, 29]. It may be further noted that the involvement of various upstream and downstream nodal pathways is characteristic of the source of cancer models [30,31]. For the first time, we have taken marginal hypoxia, which further mimics the tumor microenvironment into consideration to check its effect on an anti-cancer drug. None of the other published literature has examined environmental hypoxia, which may be detrimental or influential in the mechanism of action, especially with cancer targets. Hypoxia inducing resistance to drugs might be working though upregulation of many other pathways too. However established cell culture system with varied cancer characteristics and upon environmental selection pressure may impact our understanding of the drug targets. Henceforth, true mechanism or effect of the drug may not be studied appropriately. Consequently, progression of a potent drug or lead molecule into pre-clinical investigations may be halted.

Next, we investigated the mechanism behind the inhibition of proliferation. Apoptosis is known to be the most common method of cancer cell death [32]. Many pathways with different stimulations, stress conditions, and drugs lead cancerous cells to undergo apoptotic death [33-36]. The accumulation of early and late phaseapoptotic cells shows the efficacy of a compound to stimulate the process of apoptosis irrespective of the proliferative nature of cancer cells. Our results were in agreement and another way of confirming the induction of apoptosis by MLT-401. It is well established from the literature [37] and our own previous investigations $[17,21]$ have shown that the cells which get accumulated in the M4 fraction were apoptotic cells.

Although there are many pathways leading to apoptosis, the up-regulation of proapoptotic factors and down-regulation of anti-apoptotic factors are usually investigated to confirm the end stage apoptotic death of cancer cells. In the current investigation, MLT-401 down-regulated Bcl-2 expression, while there was not much of an up-regulation of Bax; however the ratio of Bcl-2/Bax was less than 1, which was in agreement with some standard anticancer drug evaluation studies [38-40]. Many other studies have shown that apoptosis is regulated by the anti-apoptotic molecule $\mathrm{Bcl}-2$ and relative Bax expression in different cancer models. However, the dose-dependent selective toxicity of cancer cells to MLT-401 clearly shows that this lead molecule is not affected by the hypoxic microenvironment observed in many tumor/cancer tissues. Collectively, our results indicate the mechanism of apoptotic cells death by MLT-401 and show it to be an ideal candidate lead molecule for the treatment of colorectal cancer.

\section{Conclusion}

From the above investigations, MLT-401, a novel arylidene derivative, inhibits HCT 116 colorectal cancer cells in a dose-dependent manner. Marginal hypoxia does not interfere with the anti-tumor activity of MLT-401. Apoptotic cell death is evidenced as the mechanism of cell death. A low concentration of the lead molecule is sufficient for the inhibition of cell proliferation. In conclusion, MLT-401 is an ideal candidate molecule exhibiting anticancer/ tumor activity.

\section{Acknowledgements}

The author sincerely thanks Dr. Prasanna Rajagopalan from the College of Applied Medical Science and Dr. Harish Chandramoorthy from the College of Medicine, King Khalid University, for their assistance in designing and executing this study. The author would 


\section{Cellular Physiology Cell Physiol Biochem 2018;46:2082-2089

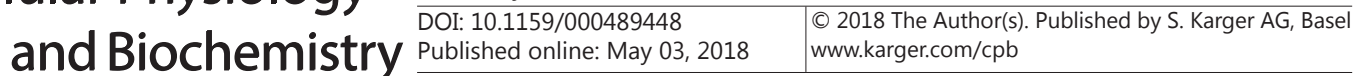 Bin-Jaliah: Hypoxia Effects on MLT-401 Anticancer Properties}

also like to express his gratitude to King Khalid University, Saudi Arabia, for providing administrative and technical support.

\section{Disclosure Statement}

No conflict of interests exists.

\section{References}

-1 Palma M, Gentilcore G, Heimersson K, Mozaffari F, Näsman-Glaser B, Young E, Rosenquist R, Hansson L, Österborg A, Mellstedt H: T cells in chronic lymphocytic leukemia display dysregulated expression of immune checkpoints and activation markers. Haematologica 2017;102:562-572.

-2 Ji LL, Sheng YC, Chen L, Wang ZT: Establishment of a new cell line for high-throughput evaluation of chemokine CCR5 receptor antagonists. Drug Discov Ther 2009;3:2-5.

-3 Quail DF, Joyce JA: Microenvironmental regulation of tumor progression and metastasis. Nat Med 2013;19:1423-1437.

-4 Palazon A, Goldrath AW, Nizet V, Johnson RS: HIF transcription factors, inflammation, and immunity. Immunity 2014;41:518-528.

5 Mak IW, Evaniew N, Ghert M: Lost in translation: animal models and clinical trials in cancer treatment. Am J Transl Res 2014;6:114-118.

6 Li F, Mei H, Gao Y, Xie X, Nie H, Li T, Zhang H, Jia L: Co-delivery of oxygen and erlotinib by aptamer-modified liposomal complexes to reverse hypoxia-induced drug resistance in lung cancer. Biomaterials 2017;145:5671.

7 Kumar H, Choi DK: Hypoxia Inducible Factor Pathway and Physiological Adaptation: A Cell Survival Pathway? Mediators Inflamm DOI: 10.1155/2015/584758.

$>8$ Wu H, Chen Q: Hypoxia activation of mitophagy and its role in disease pathogenesis. Antioxid Redox Signal 2015;22:1032-1046.

-9 Grivennikov SI, Greten FR, Karin M: Immunity, inflammation, and cancer. Cell 2010;140:883-899.

$>10$ Michiels C, Tellier C, Feron 0: Cycling hypoxia: A key feature of the tumor microenvironment. Biochim Biophys Acta 2016;1866:76-86.

11 Semenza GL: Hypoxia-inducible factors: mediators of cancer progression and targets for cancer therapy. Trends Pharmacol Sci 2012;33:207-214.

12 Sowa T, Menju T, Chen-Yoshikawa TF, Takahashi K, Nishikawa S, Nakanishi T, Shikuma K, Motoyama H, Hijiya K, Aoyama A, Sato T, Sonobe M, Harada H, Date H: Hypoxia-inducible factor 1 promotes chemoresistance of lung cancer by inducing carbonic anhydrase IX expression. Cancer Med 2017; 6 :288297.

13 SoleymaniAbyaneh H, Gupta N, Radziwon-Balicka A, Jurasz P, Seubert J, Lai R, Lavasanifar A: STAT3 but Not HIF-1 $\alpha$ Is Important in Mediating Hypoxia-Induced Chemoresistance in MDA-MB-231, a Triple Negative Breast Cancer Cell Line. Cancers (Basel) 2017;9.137 DOI: 10.3390/cancers9100137.

14 Kuo MT: Redox regulation of multidrug resistance in cancer chemotherapy: molecular mechanisms and therapeutic opportunities. Antioxid Redox Signal 2009;11:99-133.

15 Musso L, Cincinelli R, Zuco V, De Cesare M, Zunino F, Fallacara AL, Botta M, Dallavalle S: 3-ArylideneN-hydroxyoxindoles: A New Class of Compounds Endowed with Antitumor Activity. ChemMedChem 2016;11:1700-1704.

16 Sandhu HS, Sapra S, Gupta M, Nepali K, Gautam R, Yadav S, Kumar R, Jachak SM, Chugh M, Gupta MK, Suri OP, Dhar KL: Synthesis and biological evaluation of arylidene analogues of Meldrum's acid as a new class of antimalarial and antioxidant agents. Bioorg Med Chem 2010;18:5626-5633.

17 Rajagopalan P, Alahmari KA, Elbessoumy AA, Balasubramaniam M, Suresh R, Shariff ME, Chandramoorthy HC: Biological evaluation of 2-arylidene-4, 7-dimethyl indan-1-one (FXY-1): a novel Akt inhibitor with potent activity in lung cancer. Cancer Chemother Pharmacol 2016;77:393-404.

18 Bin-Jaliah I: Natural hypoxia is not a limiting factor in evaluation of MLT-40, a novel arylidene derivative, against in vitro colorectal cancer models. Proceedings of the 38th World Congress of the International Union of Physiological Sciences 2017;38:816 https://icongresso.itarget.com.br/useradm/ anais/?clt=ips.2\&id_tra=816\&imprimir $=$ tru. 


\section{Cellular Physiology Cell Physiol Biochem 2018;46:2082-2089

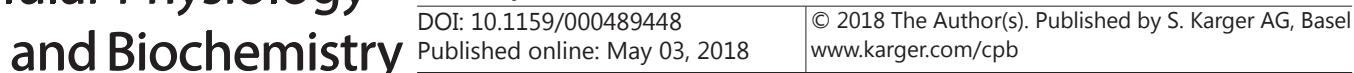

Bin-Jaliah: Hypoxia Effects on MLT-401 Anticancer Properties

19 Mancarella S, Wang Y, Deng X, Landesberg G, Scalia R, Panettieri RA, Mallilankaraman K, Tang XD, Madesh M, Gill DL: Hypoxia-induced acidosis uncouples the STIM-Orai calcium signaling complex. J Biol Chem 2011;286:44788-44798.

20 Mosmann T: Rapid colorimetric assay for cellular growth and survival: application to proliferation and cytotoxicity assays. J Immunol Methods 1983; 65:55-63.

-21 Prasanna R, Harish CC: Anticancer effect of a novel 2-arylidene-4, 7-dimethyl indan-1-one against human breast adenocarcinoma cell line by G2/M cell cycle arrest. Oncol Res 2010;18:461-468.

-22 Menezes JCJMDS: Arylidene indanone scaffold: medicinal chemistry and structure-activity relationship view. RSC Advances 2017;7:9357-9372.

23 Khloya P, Kumar P, Mittal A, Aggarwal NK, Sharma PK: Synthesis of some novel 4-arylidene pyrazoles as potential antimicrobial agents. Org Med Chem Lett 2013;3:9 DOI: 10.1186/2191-2858-3-9.

24 Stana A, Vodnar DC, Tamaian R, Pirnau A, Vlase L, Ionut I, Oniga O, Tiperciuc B: Design, Synthesis and Antifungal Activity Evaluation of New Thiazolin-4-ones as Potential Lanosterol $14 \alpha$-Demethylase Inhibitors. Int J Mol Sci 2017;18:177 DOI: 10.3390/ijms18010177.

25 Qiu X, Du Y, Lou B, Zuo Y, Shao W, Huo Y, Huang J, Yu Y, Zhou B, Du J, Fu H, Bu X: Synthesis and identification of new 4-arylidene curcumin analogues as potential anticancer agents targeting nuclear factor-kappaB signaling pathway. J Med Chem 2010;53:8260-8273.

26 Tailor NK, Boon HL, Sharma M: Synthesis and in vitro anticancer studies of novel C-2 arylidene congeners of lantadenes. Eur J Med Chem 2013;64:285-291.

27 Onnis V, Demurtas M, Deplano A, Balboni G, Baldisserotto A, Manfredini S, Pacifico S, Liekens S, Balzarini J: Design, Synthesis and Evaluation of Antiproliferative Activity of New Benzimidazolehydrazones. Molecules 2016;21

28 Mitsiades CS, Mitsiades N, Koutsilieris M: The Akt pathway: molecular targets for anti-cancer drug development. Curr Cancer Drug Targets 2004;4 :235-256.

29 Isoldi MC, Visconti MA, Castrucci AM: Anti-cancer drugs: molecular mechanisms of action. Mini Rev Med Chem 2005;5:685-695.

-30 Hassounah NB, Bunch TA, McDermott KM: Molecular pathways: the role of primary cilia in cancer progression and therapeutics with a focus on Hedgehog signaling. Clin Cancer Res 2012;18:2429-2435.

-31 Slattery ML, Herrick JS, Lundgreen A, Fitzpatrick FA, Curtin K, Wolff RK: Genetic variation in a metabolic signaling pathway and colon and rectal cancer risk: mTOR, PTEN, STK11, RPKAA1, PRKAG2, TSC1, TSC2, PI3K and Akt1. Carcinogenesis 2010;31:1604-1611.

-32 Wong RS: Apoptosis in cancer: from pathogenesis to treatment. J Exp Clin Cancer Res 2011;30:87.

33 Ramakrishnan V, D’Souza A: Signaling Pathways and Emerging Therapies in Multiple Myeloma. Curr Hematol Malig Rep 2016;11:156-164.

-34 Dillon CP, Green DR: Molecular Cell Biology of Apoptosis and Necroptosis in Cancer. Adv Exp Med Biol 2016;930:1-23.

-35 Abraha AM, Ketema EB: Apoptotic pathways as a therapeutic target for colorectal cancer treatment. World J Gastrointest Oncol 2016;8:583-591.

-36 Matsuura K, Canfield K, Feng W, Kurokawa M: Metabolic Regulation of Apoptosis in Cancer. Int Rev Cell Mol Biol 2016;327:43-87.

-37 Reyes-Reyes EM, Jin Z, Vaisberg AJ, Hammond GB, Bates PJ: Physangulidine A, a withanolide from Physalisangulata, perturbs the cell cycle and induces cell death by apoptosis in prostate cancer cells. J Nat Prod 2013;76:2-7.

38 Salakou S, Kardamakis D, Tsamandas AC, Zolota V, Apostolakis E, Tzelepi V, Papathanasopoulos P, Bonikos DS, Papapetropoulos T, Petsas T, Dougenis D: Increased Bax/Bcl-2 ratio up-regulates caspase-3 and increases apoptosis in the thymus of patients with myasthenia gravis. In vivo 2007;21:123-132.

39 Del Principe MI, Dal Bo M, Bittolo T, Buccisano F, Rossi FM, Zucchetto A, Rossi D, Bomben R, Maurillo L, Cefalo M, De Santis G, Venditti A, Gaidano G, Amadori S, de Fabritiis P, Gattei V, Del Poeta G: Clinical significance of bax/bcl-2 ratio in chronic lymphocytic leukemia. Haematologica 2016;101:77-85.

40 Vucicevic K, Jakovljevic V, Colovic N, Tosic N, Kostic T, Glumac I, Pavlovic S, Karan-Djurasevic T, Colovic M: Association of Bax Expression and Bcl2/Bax Ratio with Clinical and Molecular Prognostic Markers in Chronic Lymphocytic Leukemia. J Med Biochem 2016;35:150-157. 